\title{
Analysis and Calculation of the Fluid Flow and the Temperature Field by Finite Element Modeling
}

\author{
M. Dhamodaran, S. Jegadeesan, R. Praveen Kumar \\ Department of Electronics and Communication Engineering, M.Kumarasamy College of Engineering, Karur, Tamil Nadu, \\ 639113, India, dhamodaranm@yahoo.com
}

\begin{abstract}
This paper presents a fundamental and accurate approach to study numerical analysis of fluid flow and heat transfer inside a channel. In this study, the Finite Element Method is used to analyze the channel, which is divided into small subsections. The small subsections are discretized using higher number of domain elements and the corresponding number of nodes. MATLAB codes are developed to be used in the analysis. Simulation results showed that the analyses of fluid flow and temperature are influenced significantly by the changing entrance velocity. Also, there is an apparent effect on the temperature fields due to the presence of an energy source in the middle of the domain. In this paper, the characteristics of flow analysis and heat analysis in a channel have been investigated.
\end{abstract}

Keywords: Finite Element Method, flow analysis, temperature analysis, entrance velocity, simulation.

\section{INTRODUCTION}

The finite element method (FEM) is a numeri-cal technique to obtain an approximate solution to a class of problems governed by elliptic partial differential equations. [1]. A simple and effective FEM can be used for fluid flow and temperature flow analysis by developing a MATLAB code. The FEM is nowadays used in industrial applications, including aeronautical, aerospace, automobile, naval, and nuclear construction. A number of general-purpose computer codes are obtainable for industrial users of FEM.

The effect of FEM in the presence of a Poisson's equation for different combinations of velocity boundary conditions with prescribed fluid flow and heat flow at the boundaries has been analyzed. Such a study is beneficial in the appropriate design of many devices and methods, such as mass flow meter, ultrasonic flow meter, thermal mass flow meter, and variable area meters [2]-[4]. The resultant problem is solved using the governing equation. To complete the above goals, the paper is structured as follows. Section 2 is devoted to the numerical technique for mathematical formulation. The finite element analysis is discussed in Section 3. In Section 4 and Section 5, the numerical results presented are discussed, and some important conclusions follow in Section 6.

\section{NUMERICAL TECHNIQUE}

The goal of the numerical simulations is to determine the relationship between the transmitted energy and the changes in the flow rate of the pipe. For this numerical analysis, a pipe measuring $20 \times 50 \mathrm{~cm}$, as shown in Fig.1., is used. The fluid flows around a small pipe located in the middle of the domain with a uniform velocity $\mathrm{V}$ and having a diameter $\mathrm{d}=10 \mathrm{~cm}$ as shown in Fig.1. This small pipe contains an energy source that produces $q$ units of energy per surface area of the pipe per unit time.

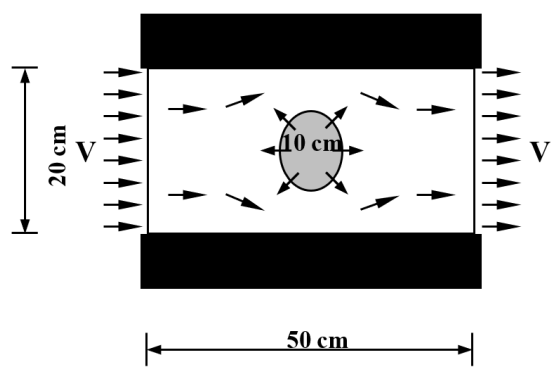

Fig.1. Pipe flow system.

The fluid flow is solved using the stream function for a specific value of entrance velocities [5]. Once the flow is determined, the temperature field can be established by solving the temperature governing equation [6].

The governing equation that governs the two-dimensional problem, in general can be obtained from the below relation:

$$
\begin{array}{r}
\frac{\partial}{\partial x}\left(R_{x} \frac{\partial \Phi}{\partial x}\right)+\frac{\partial}{\partial y}\left(R_{y} \frac{\partial \Phi}{\partial y}\right)+B_{x} \frac{\partial \Phi}{\partial x}+B_{y} \frac{\partial \Phi}{\partial y}+G \Phi+H=0 \text { in } \Omega \\
\Phi=\Phi^{*} \text { on } S_{1} \\
R_{x} \frac{\partial \Phi}{\partial x} n_{x}+R_{y} \frac{\partial \Phi}{\partial y} n_{y}=q^{*} \text { on } \mathrm{S}_{2}
\end{array}
$$


The coefficients $R_{x}, R_{y}, B_{x}, B_{y}, G$, and $H$ are given as functions of $\mathrm{x}$ and $\mathrm{y}$. The two-dimensional domain is denoted by $\Omega . S$ is the boundary of the domain, and $S_{1}$ and $S_{2}$ are parts of that boundary. $\mathrm{n}_{\mathrm{x}}$ and $\mathrm{n}_{\mathrm{y}}$ represent the outward normal unit vectors on the boundary [7]-[11].

Poisson's equation originates from the general governing partial differential equation, and it is adequate to describe a large number of applied problems including the flow of ideal fluids problems. The two governing equations for the flow of ideal fluids are shown below:

$$
\begin{aligned}
& \frac{\partial u_{x}}{\partial y}-\frac{\partial u_{y}}{\partial x}=0 \\
& \frac{\partial u_{x}}{\partial x}+\frac{\partial u_{y}}{\partial y}=0
\end{aligned}
$$

If we define $\Psi$ such that it identically satisfies the condition for incompressible flow, then we have

$$
\begin{aligned}
& u_{x}=+\frac{\partial}{\partial y} \Psi \\
& u_{y}=-\frac{\partial}{\partial x} \Psi
\end{aligned}
$$

By substituting equations (4) and (5) into the equation (2), the stream function of the fluid flow can be written as follows:

$$
\frac{\partial^{2} \Psi}{\partial x^{2}}+\frac{\partial^{2} \Psi}{\partial y^{2}}=0
$$

For steady state two-dimensional convection through a constant-property homogenous fluid, the energy equation is given by [7]

$$
\frac{\partial}{\partial x}\left(\rho C_{p} u_{x} \varphi-k \frac{\partial \varphi}{\partial x}\right)+\frac{\partial}{\partial y}\left(\rho C_{p} u_{y} \varphi-k \frac{\partial \varphi}{\partial y}\right)=0
$$

The above equation can also be rewritten as follows:

$$
\frac{\partial}{\partial x}\left(k \frac{\partial \varphi}{\partial x}\right)+\frac{\partial}{\partial y}\left(k \frac{\partial \varphi}{\partial y}\right)-\rho C_{p} u_{x} \frac{\partial \varphi}{\partial x}-\rho C_{p} u_{y} \frac{\partial \varphi}{\partial y}=0
$$

Here, $\varphi$ is the temperature, $k$ is the thermal conductivity of the fluid, and $\rho C_{P}$ is the heat capacity of the fluid. $u_{x}$ and $u_{y}$ denote the velocity components in $\mathrm{x}$ and $\mathrm{y}$ directions, respectively. Since the time-dependency is beyond the scope of this paper, the thermal conductivity of the fluid will be assumed to be time-independent and take a constant value $k=1.0$. Also, the heat capacity $\rho C_{P}$ is assumed to be equal to 1.0 .
In this paper, a two-dimensional steady flow problem is solved using the finite element technique. The flow field of that fluid then resolves the partial differential equations of temperature flow. For both fluid flow and temperature field, boundary conditions are applied. The domain of the problem is discretized to a large number of elements to promise the exactness of the solution. Both the fluid flow and temperature field have been reviewed for various values of entrance velocity.

\section{FINITE ELEMENT ANALYSIS}

The finite element analysis obtains the temperatures, stresses, flows, or other desired unknown parameters in the FEM by minimizing the energy functional. The energy functional consists of all the energies associated with the particular FEM. Based on the law of conservation of energy, the finite element energy functional must equal to zero. For the numerical simulation of a pipe, MATLAB code using a mesh generator is developed [12]-[14].

A scheme of a main, typical module with discretization is shown in Fig.2. The finite element technique involves dividing the analysis region into several sub-regions. These small regions are the elements, which are linked with adjacent elements at their nodes. Mesh generation is a process of generating the geometric data of the elements and their nodes, and involves computing the coordinate nodes, defining their connectivity, and thus, constructing the elements. Here, mesh designates aggregates of elements, nodes and lines representing their connectivity. Capability and convenience of modeling the analysis domain are dominated by the mesh generation procedure. The geometric characteristics of generated elements affect the overall performance and accuracy of the finite element analysis. Therefore, mesh generation is one of the most important procedures in FEM.

The input data for the mesh generator include the number of generation loops and some geometric coordinates of specific points on each side of each loop. Thus, the total used number of elements is discretized into domain element to maintain the continuity of the degrees of freedom along the edges of the elements. It should be noted, as shown in Fig.3., that the sizes of the elements decrease on getting closer to the small pipe in the middle so as to ensure the accuracy of the solution in this area of concentrated stresses. The mesh discretization is provided in both the flow and temperature analyses.

A problem encountered with the two-dimensional problem analyses is how to number the nodes such that it minimizes the storage needed in the stiffness matrix. The bandwidth of the stiffness matrix depends on the way the nodes have been numbered. Also, the difference between a good numbering scheme and a poor numbering scheme can result in a very large difference in bandwidth requirements. Since finite element equations are related to each other only through common elements, the reduction of the bandwidth needs nodes that are connected by common elements that are as close in numerical value as possible. 


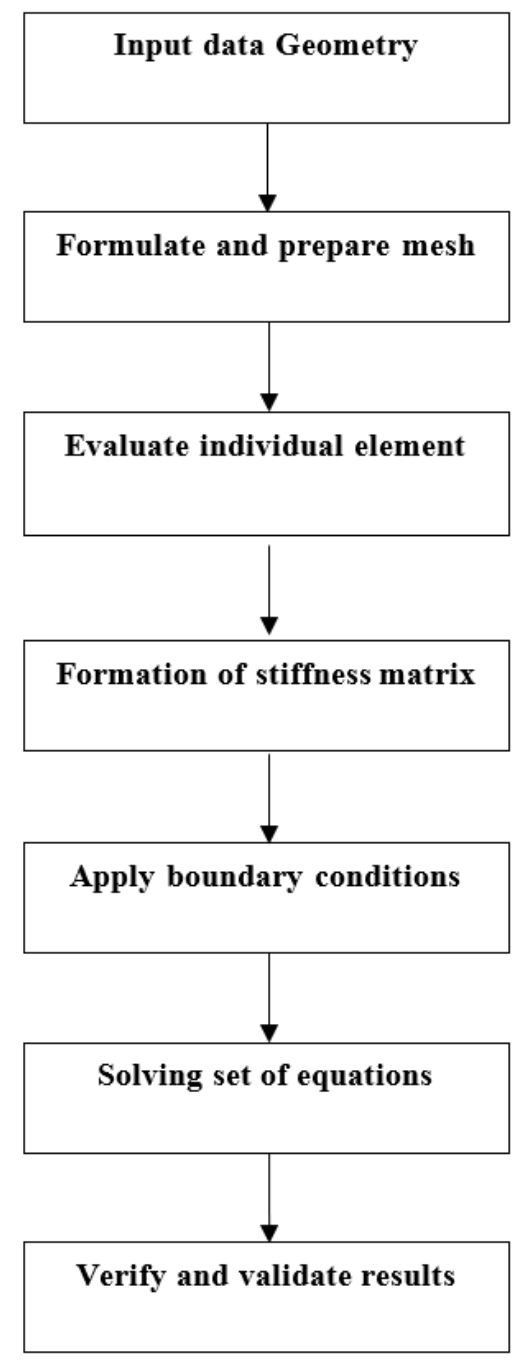

Fig.2. FEM flowchart.

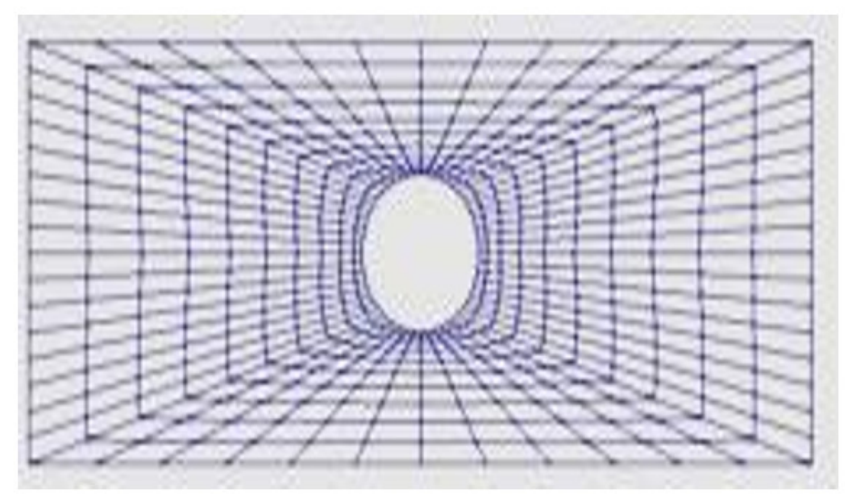

Fig.3. Mesh discretization.

The line connecting these nodes is referred to as the first wave of nodes, as shown in Fig.4. The second wave consists of all nodes that link to nodes in the first wave through common elements. The nodes in the second wave are then given the next consecutive numbers in the new order. This process continues until all nodes have been given new numbers. Finally, all elements should have node numbers that differ by no more than the number of nodes in the lengthy two consecutive waves, and even less. The output contains the new numbering scheme that will be used in the finite element analysis.

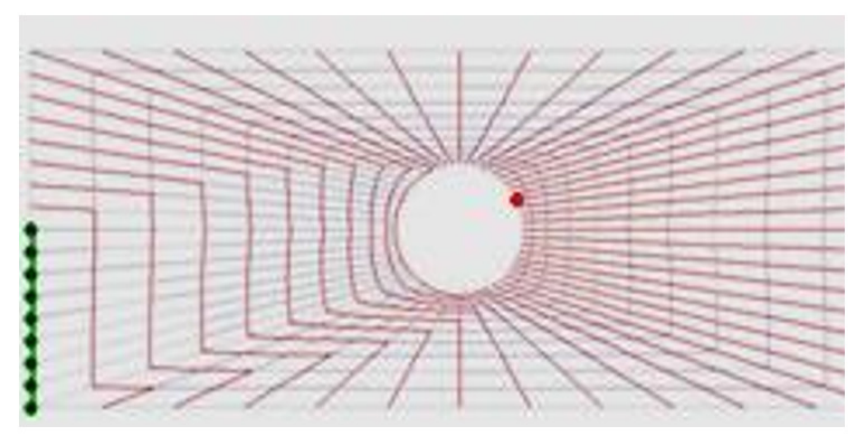

Fig.4. Wave of nodes.

\section{FLOW ANALYSIS}

Both fluid flow and temperature field can be described by the general equation that governs the two-dimensional problems. However, the coefficients of the governing equation (1) differ from the fluid flow to the temperature field such that they satisfy the governing equations given in equations (6) and (8) for fluid flow and temperature flow, respectively.

Since the flow analysis is characterized by the Poisson's expression; it is recalled here for convenience.

$$
\frac{\partial^{2} \Psi}{\partial x^{2}}+\frac{\partial^{2} \Psi}{\partial y^{2}}=0
$$

The boundary conditions for the fluid flow analysis are illustrated in Fig.5. The x-component of the velocity at the entrance and the exit is constant. $u_{x}$ is constant specific value for the velocity and $y$ is the $y$-coordinate of the nodes in that boundary. Since the flow is non-viscous and incompressible, the x-component of the velocity remains constant at the upper and lower boundaries. Thus, the boundary conditions of fluid flow at upper and lower boundaries can be given by the following relations:

$$
\begin{gathered}
\Psi=u_{x}(10) \\
\Psi=u_{x}(-10)
\end{gathered}
$$

The values 10 and -10 are the $y$-coordinates of the nodes on upper and lower boundaries, respectively. The y-component of the velocity, i.e., $\mathrm{u}_{\mathrm{y}}$ is constant and equal to zero. Also, it is maintained the non-viscosity and incompressibility of the fluid. Once the boundary conditions and the coefficients are defined, the analysis of the fluid flow is determined. Results are attained for altered values of the velocity $u_{x}=0.0,0.40$, 0.80 , and 1.0 . 


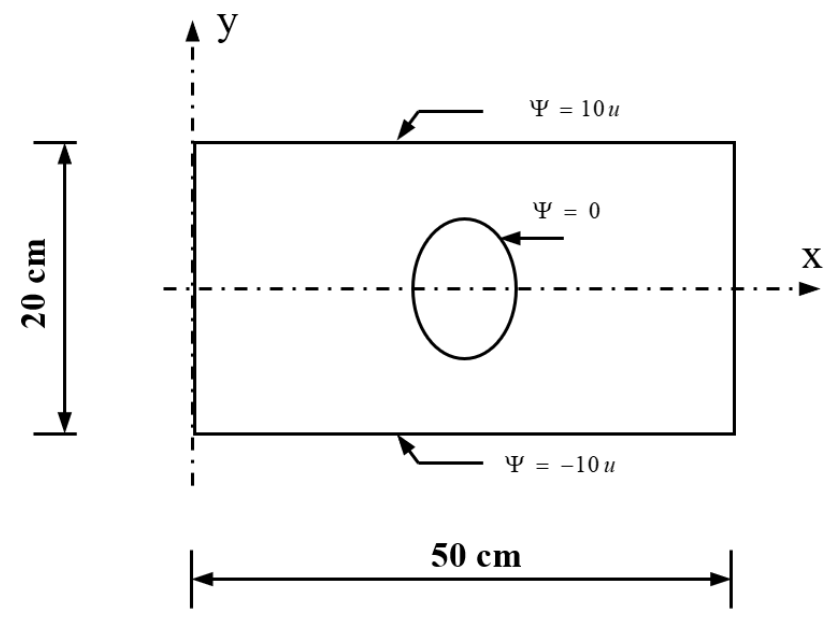

Fig.5. Boundary conditions of the fluid flow.
Fig.6. shows the fluid flow of the different specified values of the entrance velocity. As illustrated in the results, the flow field is the same for all values of the velocities; however, the absolute value of the flow increases with increasing entrance velocity. Also, it is noticed that when the velocity $u_{x}=0.0$, the flow field is static and the stream function is constant all over the domain.

The temperature flow is characterized by the energy equation given by equation (8). Here, the coefficients of the general governing equation (1), $\mathrm{R}_{\mathrm{x}}, \mathrm{R}_{\mathrm{y}}, \mathrm{B}_{\mathrm{x}}, \mathrm{B}_{\mathrm{y}}, \mathrm{G}$, and $\mathrm{H}$, take the following values; $R_{x}=k, R_{y}=k, B_{x}=-\rho C_{P u_{x}}, \quad B_{y}=-$ $\rho \mathrm{C}_{\mathrm{Pu}}, \mathrm{G}=0.0$, and $\mathrm{H}=0.0$. The thermal conductivity $k$ and the heat capacity of the fluid $\rho C_{P}$ are assumed to have unit values. The coefficients $B_{x}$ and $B_{y}$ are functions of velocity components $\mathrm{u}_{\mathrm{x}}$ and $\mathrm{u}_{\mathrm{y}}$, respectively. The velocity components $\mathrm{u}_{\mathrm{x}}$ and $\mathrm{u}_{\mathrm{y}}$ are given by using equations (4) and (5) as derivatives of the fluid flow with respect to $\mathrm{x}$ and $\mathrm{y}$, respectively.
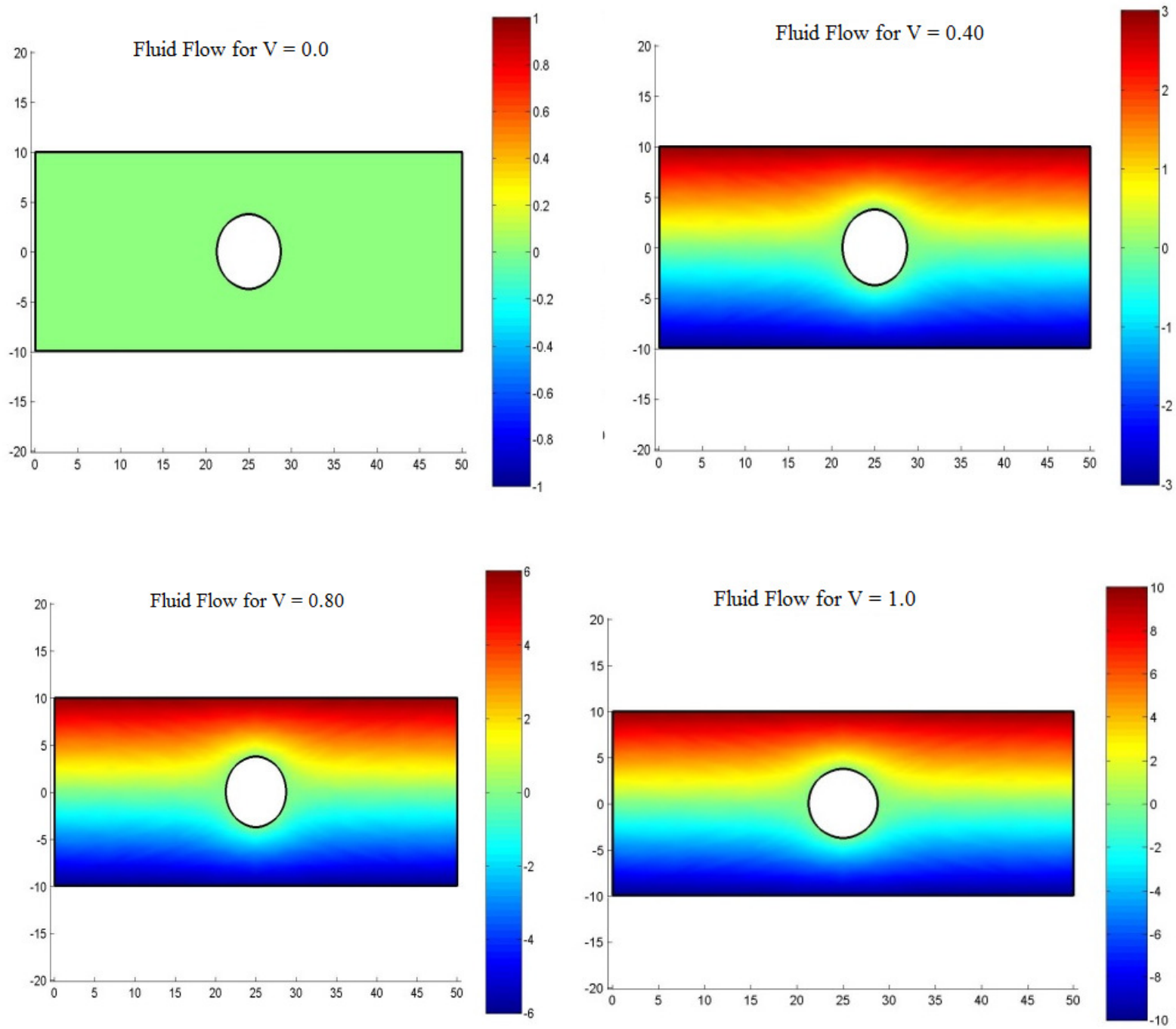

Fig.6. Fluid flow for different values of entrance velocity. 


\section{TEMPERATURE ANALYSIS}

The boundary conditions for the temperature field analysis are illustrated in Fig.7. The small pipe in the middle is assumed to produce $q$ units of energy per surface area of the pipe per unit time. The energy $q$ is considered equal to unity at the small pipe and equal to zero at exit, lower boundary and upper boundary. The temperature flow $\varphi$ is considered equal to zero at the entrance. Once the boundary conditions and the coefficients are defined, the analysis of the temperature field is ascertained.

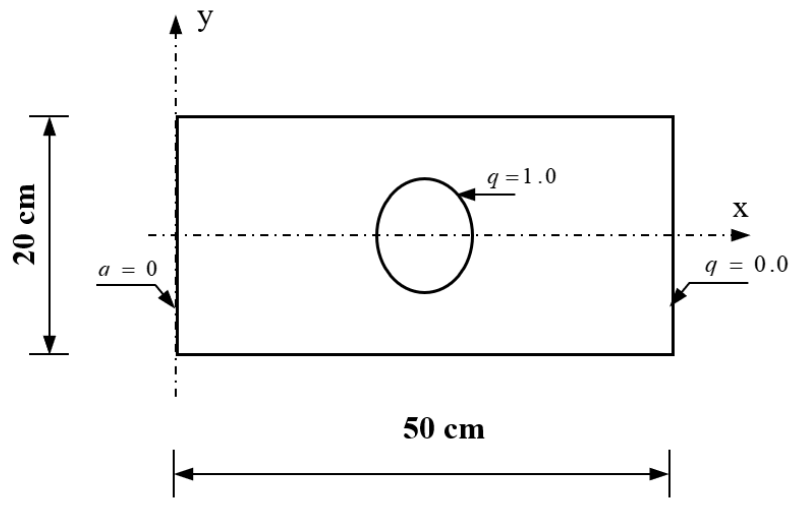

Fig.7. Boundary conditions of the temperature field.

Results are attained for altered values of the velocity, $\mathrm{u}_{\mathrm{x}}=$ $0.0,0.40,0.80$, and 1.0. Fig.8. shows the flow field of the temperature at different specified values of the entrance velocity. It is noticed from the results that there is a steep decrease in the temperature field with increasing entrance velocity. This coincides with the fact that the heat transferred by convection is greater than heat transferred by conduction. Also, Fig.8. illustrates that the rate of heat transferred through the domain is increasing with increasing velocity, which results in a decrease in temperature near the heat source in the middle of the domain.

In the present study, the flow is considered to be twodimensional with no difference in the span-wise direction. A partial differential equation is used to represent the flow domain. A series of numerical calculations have been conducted, and the results are presented in order to show the effects of fluid flow and temperature field on temperature distribution in the pipe channel. It should be noted that since the pipe size in the flow direction is relatively coarse, the local heat transfer is not as accurate or detailed as is the case of the $\mathrm{x}$ and $\mathrm{y}$ directions. However, the resolution is sufficient to aid in the design of pipe for industrial applications and also to provide information and insight into the fluid flow characteristics in the flow direction. In reality, since it is difficult to achieve an adiabatic boundary at the inlet and outlet of the pipe as assumed in the numerical model, a significant portion of the heat loss is transferred to the ambient environment, especially for low fluid flow conditions. Thus, when evaluating the heat transfer in pipe with low fluid flow rates, particular attention should be paid to the effects of this heat loss.
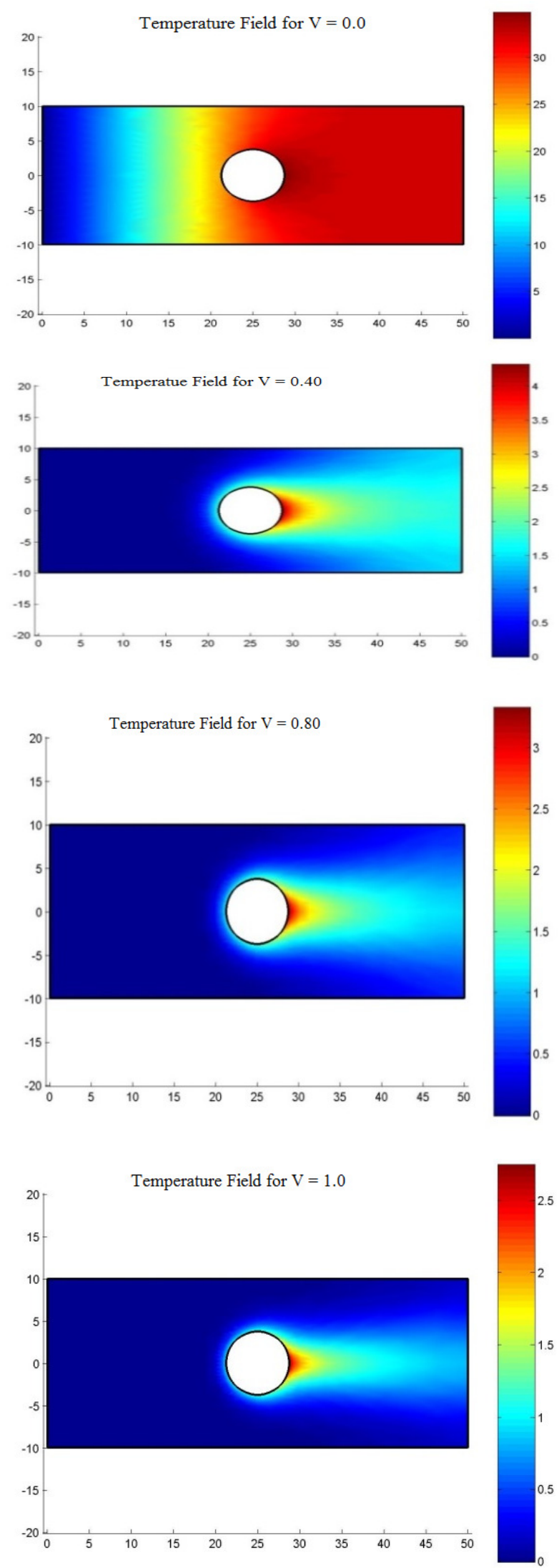

Fig.8. Temperature field for different values of entrance velocity. 


\section{CONCLUSIONS}

In this paper, the numerical simulation of fluid flow and temperature field in a pipe channel for different entrance velocity is analyzed using the FEM through solving partial differential equations of the fluid flow. The fluid flow is expressed by the partial differential equation. The heat transfer is analyzed using the energy equation. The flow field of that fluid is then used to solve the partial differential equations of temperature flow. The flow structure and heat transfer characteristics are studied in detail.

A fundamental and accurate technique is used to compute the steady flow problem using the FEM. The formulation is based on the differential equation and the energy equation. The obtained results show that the fluid flow is characterized by a circulation upwards and that the presence of a mode of heat transfer is purely conductive. The effect of the presence of heat source inside the field of the temperature flow is also inspected. According to these results of this work, we can conclude that our calculation seems to be in concordance with some other works found in the literature for simple geometries. However, the simplicity of the measurement techniques makes FEM a suitable method for steady flow analysis.

\begin{tabular}{|l|}
\hline NOMENCLATURE \\
\hline $\mathrm{x}, \mathrm{y}$ - Cartesian coordinates \\
\hline$\Psi$ - temperature \\
\hline$k$ - thermal conductivity \\
\hline$S$ - the boundary of the domain \\
\hline $\mathrm{S}_{1}, \mathrm{~S}_{2}-$ part of the boundary \\
\hline$\rho \mathrm{C}_{\mathrm{P}}$ - heat capacity \\
\hline $\mathrm{q}$ - energy source \\
\hline $\mathrm{V}$ - velocity \\
\hline $\mathrm{d}$ - diameter \\
\hline $\mathrm{R}_{\mathrm{x}}, \mathrm{R}_{\mathrm{y}}, \mathrm{B}_{\mathrm{x}}, \mathrm{B}_{\mathrm{y}}, \mathrm{G}, \mathrm{H}-$ coefficients \\
\hline $\mathrm{u}_{\mathrm{x}}-$ velocity of the $\mathrm{x}$ component \\
\hline $\mathrm{u}_{\mathrm{y}}-$ velocity of the y component \\
\hline
\end{tabular}

\section{REFERENCES}

[1] Dhamodaran, M., Dhanasekaran, R. (2014). Comparison of computational electromagnetics for electrostatic analysis. International Journal of Energy Optimization and Engineering, 3 (3), 86-100.

[2] Raine, A.B., Aslam, N., Underwood, C.P., Danaher, S. (2015). Development of an ultrasonic airflow measurement device for ducted air. Sensors, 15 (5), 10705-10722.
[3] Daev, Z.A. (2015). A comparative analysis of the discharge coefficients of variable pressure-drop flowmeters. Measurement Techniques, 58 (3), 323326.

[4] Jiang, W., Zhang, T., Xu, Y. et al. (2016). The effects of fluid viscosity on the orifice rotameter. Measurement Science Review, 16 (2), 87-95.

[5] Finlayson, B.A. (1970). Convective instability of ferromagnetic fluids. Journal of Fluid Mechanics, 40, 753-767.

[6] Baker, R.C. (2004). The impact of component variation in the manufacturing process on variable area (VA) flowmeter performance. Flow Measurement and Instrumentation, 15 (4), 207-213.

[7] Guiggiani, M. (1999). The evaluation of Cauchy principal value integrals in the boundary element method--a review. Mathematical and Computer Modelling, 15 (3), 175-184.

[8] Schena, E., Massaroni, C., Saccomandi, P., Cecchini, S. (2015). Flow measurement in mechanical ventilation: A review. Medical Engineering \& Physics, 37 (3), 257264.

[9] Ning, J., Peng, J. (2009). A temperature compensation method based on neural net for metal tube rotameter. In International Conference on Transportation Engineering 2009. ASCE, 2334-2339.

[10] Turkowski, M. (2004). Influence of fluid properties on the characteristics of a mechanical oscillator flowmeter. Measurement, 35 (1), 11-18.

[11] Turkowski, M. (2003). Progress towards the optimization of a mechanical oscillator flowmeter. Flow Measurement and Instrumentation, 14 (1-2), 1321.

[12] Gong, Y., Liu, Q.F., Zhang, C.L., Wu, Y., Rao, Y.R., Peng, G.D. (2015). Microfluidic flow rate detection with a large dynamic range by optical manipulation. IEEE Photonics Technology Letters, 27 (23), 25082511.

[13] Sadiku, M.N.O. (2007) Elements of Electromagnetics, 4th Edition. Oxford University Press, 740-748.

[14] Thompson, E.G. (2004) Introduction to the Finite Element Method: Theory Programming and Applications. John Wiley \& Sons.

[15] Jin, J. (2002) Finite Element Method in Electromagnetics. Wiley.

Received September 12, 2017. Accepted March 20, 2018. 